\title{
Cystic fibrosis carrier screening: Validation of a novel method using BeadChip technology
}

Lisa Edelmann, $P h D^{1}$, Ghazala Hashmi, $P h D^{2}$, Yuhua Song, $M S^{2}$, Yi Han, $M S^{2}$, Ruth Kornreich, $P h D^{1}$, and Robert J. Desnick, PhD, $M D^{1}$

\begin{abstract}
Purpose: To validate a novel BeadChip assay system for cystic fibrosis (CF) mutation testing using the panel of 25 ACMG recommended mutations and $\mathrm{D} 1152 \mathrm{H}$. Methods: DNA from 519 individuals originally tested for CF mutation status by allele specific oligonucleotide hybridization (ASOH) were blindly analyzed by the BeadChip assay and the results were compared. The elongation mediated multiplexed analysis of polymorphisms (eMAP) protocol, which combines multiplex amplification of genomic DNA and multiplex detection of mutations on color-coded bead arrays, was used to analyze 26 CF mutations in two separate groups. Results: The system accurately distinguished the 26 CF genotypes and had $100 \%$ concordance with the ASOH technique with an assay failure rate of $1.7 \%$. Benign variants of exon 10 codons 506,507 , and 508 did not interfere with mutation identification and reflex testing for the 5/7/9T IVS8 polymorphism was performed on a separate array. Conclusions: The BeadChip assay system provided accurate and rapid identification of the ACMG recommended CF mutations. Genet Med 2004:6(5):431-438.
\end{abstract}

Key Words: cystic fibrosis, carrier screening, BeadChip technology

Cystic fibrosis (CF) results from mutations in the CF transmembrane conductance regulator (CFTR) and is a common autosomal recessive disorder, particularly in individuals of Caucasian and Ashkenazi Jewish (AJ) ancestry. ${ }^{1,2}$ CF also affects individuals from other ethnic groups, including Hispanics, African Americans, and Asians with carrier frequencies ranging from 1 in 46 to 1 in $90 .{ }^{1}$ More than 1000 mutations have been described in the CFTR gene and although many of them are private mutations, there are a number of mutations that are distributed worldwide and still others that are common to specific ethnic groups. ${ }^{3}$ In 2001, the American Colleges of Medical Genetics (ACMG) and Obstetrics and Gynecologists (ACOG) established guidelines for prenatal carrier testing for $\mathrm{CF}$ that included a panel of 25 panethnic mutations with allele frequencies $\geq 0.1 \%$ among CF patients in North America. ${ }^{1,4}$ In addition, they recommended that carriers of R117H be subsequently tested for the 5/7/9T polymorphic alleles in intron 8 and that individuals positive for delF508 and delI507 have reflex testing for interference from the benign variants F508C, I506V, and I507V. ${ }^{1}$

The ACMG/ACOG recommendations precipitated a dramatic increase in the number of CF tests performed in genetic testing laboratories. In addition, 11 states have added CF to their newborn screening program as a two-tier process, which includes an immunoreactive trypsinogen assay followed by

\footnotetext{
From the ${ }^{1}$ Department of Human Genetics, Mount Sinai School of Medicine, New York, New York; and ${ }^{2}$ BioArray Solutions Ltd, Warren, New Jersey.

Robert J. Desnick, PhD, MD, Professor and Chairman, Department of Human Genetics, Mount Sinai School of Medicine, Box 1498, Fifth Ave at 100th St, New York, NY 10029.

Received: May 13, 2004.

Accepted: June 11, 2004.
}

DOI: 10.1097/01.GIM.0000140836.66050.88 mutation analysis as reflex testing and confirmation..$^{5-7}$ Thus, the rise in requests for $\mathrm{CF}$ testing necessitates the development of rapid, robust, and efficient assay methods.

A variety of techniques have been used by genetic testing laboratories for mutation detection of $\mathrm{CF}$ alleles that include the ACMG recommended 25 mutations. Some laboratories have developed CF testing in-house, whereas others use commercially available Analyte Specific Reagents (ASRs) as well as specialized instrumentation. Although most testing methods require multiplexed PCR for DNA amplification, the technologies used to differentiate wild type from mutant alleles after PCR vary and include allele specific oligonucleotide hybridization $(\mathrm{ASOH})$, reverse dot blot (RDB), oligonucleotide ligation assay (OLA-PCR) and amplification refractory mutation system (ARMS), among others. Each of these methods is widely used and can genotype CF alleles; however, a number of drawbacks have been noted. ${ }^{2,8,9}$ The ASO method is a home-brew and, therefore, has no commercial ASRs, and although it can be semiautomated with a robotic workstation, ${ }^{10}$ it is labor intensive and in most cases uses radioactive isotopes. In addition, the ASO method is hybridization-based and in order to reduce labor and materials, uses pooled probe groups that are tested individually only after a positive is identified within a group. The RDB is available commercially with ASRs but is relatively inflexible with respect to the mutation panel. OLA-PCR has commercial ASRs; however, expensive equipment is required for analysis and is, therefore, more feasible for larger laboratories. The ARMS method is also available as a commercial kit with ASRs, but will not distinguish most homozygous genotypes, and therefore requires additional methods for reflex testing of positive samples. Currently, laboratories that perform CF mutation analysis have several existing options and 
use the testing method that best suits their needs. However, as new technologies emerge, those that are robust, rapid, and cost-effective are likely to replace existing methods.

During the past seven years, our laboratory has performed mutation detection for various genetic diseases by multiplex PCR followed by ASOH. This home-brew methodology has been used for CF testing on over 60,000 samples. In this study, we report the validation of a new semiautomated method using a novel BeadChip technology with the eMAP (elongation mediated multiplexed analysis of polymorphisms) protocol developed at BioArray Solutions Ltd. (BAS) for the rapid, sensitive, and reproducible detection of the ACMG recommended CF panel. ${ }^{1}$ The eMAP protocol combines multiplex amplification of genomic DNA and multiplex detection of mutations and polymorphisms using ASOs with variable 3'-terminal sequences displayed on color-encoded beads that are assembled into random arrays on semiconductor chips. After amplification, single-stranded PCR products are annealed to the beaddisplayed ASOs that function as allele-specific forward primers. These are elongated when there is complementarity with the annealed amplicon and are rendered visible by incorporation of a fluorescently labeled nucleotide analog. Elongation products of the panel of mutations and wild-type sequences are simultaneously detected by instant imaging of the entire array using an automated Array Imaging System (AIS) also provided by BAS. The BeadChip format facilitates customization, ensures rapid, and exceptional discrimination of wild type and mutant alleles, and provides automated allele calling with electronic data storage. In this study, we describe the validation of this BeadChip assay system for CF carrier detection in the Genetic Testing Laboratory at Mount Sinai School of Medicine (MSSM).

\section{METHODS}

\section{Patient population and control samples}

The BeadChip assay system and elongation-mediated multiplexed analysis of polymorphisms (eMAP) protocol were evaluated using DNA samples of 507 patients and 12 proficiency samples referred to our laboratory for CF screening. CF testing was initially performed by ASOH and results were reported within two weeks after receipt of the sample. After reporting the test results, DNA from these samples were tested for CF using the eMAP protocol and BeadChip assay system. Mutation controls included DNA from previously identified positive patient samples (I148T, D1152H, W1282X, R117H, G85E, A455E, delF508, N1303K) and DNA from NIGMS Human Genetic Cell Repositories (Coriell Cell Repositories) (delF508, delI507, G542X, R560T, 3849+10kbC > T, N1303K, G85E; G551D, R553X, 621+1G>T, 1717-1G>A, A455E, R334W, R347P, R1162X, 3659delC; 711+1G > T, 2789+5G >A, $3120+1 \mathrm{G}>\mathrm{A}$ ). Controls for the rare mutations 1078delT, $1898+1 \mathrm{G}>\mathrm{A}$, and 2184delA were reamplified from PCR products obtained from the respective heterozygous individuals. All patient samples that were used as positive controls were confirmed by sequencing. Normal controls were included in each run.

\section{DNA purification}

Genomic DNA was extracted from peripheral blood samples using the simple and rapid lysis method as previously described. ${ }^{11}$ All patient DNA were tested for CF with informed consent and the study was conducted with IRB approval at MSSM.

\section{Allele-specific oligonucleotide hybridization}

Multiplex PCR analysis was performed in two reactions with seven amplimers in Group I and nine amplimers in Group II for the analysis of the ACMG panel of 25 mutations plus D1152H. Group I amplimers included CFTR exons 3, 4, 10, 11, 20, 21, and intron 19. Group II amplimers included CFTR exons $5,7,9,12,13,14 \mathrm{~b}, 16 \mathrm{a}, 18$, and 19. All primers contained a 20 nucleotide universal primer sequence at the 5 ' end and were amplified as described elsewhere. ${ }^{12}$ Patient and control samples for all 26 mutations were included with each run. Fifteen microliters of all PCR products were visualized by electrophoresis on $2 \%$ agarose gels to evaluate amplification quality. Group I multiplex PCR reactions were spotted onto three Hybond-N+ membranes, and Group II multiplex PCR reactions were spotted to four $8 \times 12 \mathrm{~cm}$ Hybond-N+ membranes (Amersham Pharmacia Biotech), using the Biomek 2000 automated workstation (Beckman Coulter). Mutant ASOs were end-labeled with $\gamma^{32} \mathrm{P}$-ATP and pooled into three subgroups (IA-IC) for Group I and four subgroups (IIA-IID) for Group II mutations with the following breakdown of mutations: IA: delF508, delI507, W1282X, R117H; IB: G542X, R560T, $3849+10 \mathrm{kbC}>\mathrm{T}, \mathrm{N} 1303 \mathrm{~K}, \mathrm{G} 85 \mathrm{E} ; \mathrm{IC}: \mathrm{G} 551 \mathrm{D}, \mathrm{R} 553 \mathrm{X}$, $621+1 \mathrm{G}>\mathrm{T}, 1717-1 \mathrm{G}>\mathrm{A}, \mathrm{I} 148 \mathrm{~T}$; IIA: A455E, R334W, D1152H; IIB: R347P, 1078delT, R1162X, 3659delC; IIC: $711+1 \mathrm{G}>\mathrm{T}, 1898+1 \mathrm{G}>\mathrm{A}, 2789+5 \mathrm{G}>\mathrm{A}, 3120+1 \mathrm{G}>\mathrm{A} ; \mathrm{IID}:$ 2184delA. Hybridizations were performed in TMAC hybridization buffer with 10-fold molar excess of unlabeled WT oligos as competitor at $55^{\circ} \mathrm{C}$ for 2 hours to $\mathrm{O} / \mathrm{N} .{ }^{13}$ Filters were washed twice at $55^{\circ} \mathrm{C}$ for 15 minutes in TMAC wash buffer and exposed to Biomax MR film (Kodak). ${ }^{13}$

Reflex testing for positives was performed by amplification of reextracted DNA and hybridization with individual mutant and wild-type oligonucleotide probes for each mutation within the group. Reflex testing for the 5T/7T/9T variants of the intron 8 polypyrimidine tract was performed for all R117H-positive samples. Reflex testing of delF508 and delI507 positive samples for the F508C, I506T, and I507T variants was not necessary with this methodology.

\section{BeadChip assay system}

All BeadChip assay components were provided by BAS. Each of the runs for validation of the BeadChip assay system, I through VIII, was performed subsequent to the report of CF results assayed by the ASOH method. Each run (I-VIII) corresponds to a set of samples that were initially tested for CF by ASOH. All 26 CF mutations (52 probes) were tested on all samples within each run. Because each sample was amplified in two separate multiplex PCR reactions, there were two distinct BeadChips used for each sample, one that contained the nor- 
mal and mutant probes for GPI mutations and another that contained the normal and mutant probes for GPII mutations. Controls were run with each amplification, except for run I, which was amplified simultaneously with run II and with the same master mixes. A subset of the mutation controls were included with each run with the majority of mutations represented in each run with a total of 145 mutation control samples assayed in total. Overall, each mutation was assayed in the heterozygous state at least 3 times. Homozygous samples were included in the analysis for delF508 and 2789+5G $>$ A.

\section{Elongation-mediated multiplexed analysis of polymorphisms (eMAP)}

BAS's eMAP protocol for the analysis of CF genotypes, using the BeadChip assay system, involves multiplex PCR amplification, strand selection, and multiplex elongation. The individual steps are illustrated in Fig. 1A and described later.

\section{Multiplex PCR amplification}

Genomic DNA was amplified with corresponding primers in two separate multiplex PCR reactions (Groups I and II) using a GeneAmp 9700 thermal cycler (ABI) with an initial "hot start" polymerase activation step at $94^{\circ} \mathrm{C}$ for 15 minutes followed by 30 cycles, each composed of denaturation for 30 seconds at $94^{\circ} \mathrm{C}$ with a $60 \%$ ramp, annealing for 30 seconds at $60^{\circ} \mathrm{C}$ with a $50 \%$ ramp, and extension for 20 seconds at $72^{\circ} \mathrm{C}$ with a $35 \%$ ramp followed by extension for 8 minutes at $72^{\circ} \mathrm{C}$. Each reaction $(25 \mu \mathrm{L})$ contained $\approx 200 \mathrm{ng}$ genomic DNA, $1 \times$ PCR buffer $(10 \mathrm{mmol} / \mathrm{L}$ Tris-HCL, $50 \mathrm{mmol} / \mathrm{L} \mathrm{KCL}, 0.1 \%$ Triton X-100), $1.5 \mathrm{mmol} / \mathrm{L} \mathrm{MgCl}_{2}, 200$ $\mu \mathrm{mol} / \mathrm{L}$ each of PCR grade dNTPs, and 5 units of Hot Star Taq DNA polymerase (Qiagen). Group I amplification included exons $3,4,9,10,11,20,21$, and intron 19 and group II included exons $5,7,12,13,14 \mathrm{~B}, 16,18$, and 19 . To permit strand selection ("+" or "-" strand), the forward primers for exons 7 and 10 and the reverse primer(s) for all other amplicons, were modified by 5 ' $\mathrm{OH}$-phosphorylation.

\section{Post-PCR processing}

Aliquots $(6.5 \mu \mathrm{L})$ of PCR product were processed in a new tube to remove excess primers and dNTPs using ExoSAPIT (Amersham Pharmacia Biotech). To produce single-stranded DNA templates, PCR products were incubated in the GeneAmp 9700 thermal cycler (ABI) with 2.5 units of $\lambda$ exonuclease (Amersham Pharmacia Biotech) in $1 \times$ buffer at $37^{\circ} \mathrm{C}$ for 20 minutes; the enzyme was then inactivated at $75^{\circ} \mathrm{C}$ for 10 minutes.

\section{On-chip elongation}

Single-stranded amplicons $(10 \mu \mathrm{L})$ were added to an equal amount of elongation mixture containing $3 \mathrm{U}$ of Thermo Sequenase (Amersham Pharmacia Biotech), $1 \times$ enzyme buffer with TAMRA-labeled deoxycytosine (dCTP) analog (NEN Life Sciences), and $1 \mu \mathrm{mol} / \mathrm{L}$ of each unlabeled dNTP. Each elongation sample $(20 \mu \mathrm{L})$ was transferred to a single BeadChip mounted on an 8 chip carrier $(25 \times 75 \mathrm{~mm})$. The elongation reaction was allowed to proceed for 15 minutes at $53^{\circ} \mathrm{C}$ and 3 minutes at $60^{\circ} \mathrm{C}$ in a temperature-controlled BeadChip processor developed by BAS that held four slides each with eight BeadChips.
After elongation, BeadChips were washed three to four times with $\mathrm{ddH} 2 \mathrm{O}$. An image of the fluorescence pattern of beads within the array was recorded using an automated Array Imaging System (BAS) equipped with fluorescence optics and a CCD camera. Images were analyzed using ImageStudio software (BAS) by determining the identity of elongated probes.

\section{BeadChip design}

Allele specific probes (20-25 bp) for $26 \mathrm{CF}$ mutations were designed with $30 \%$ to $50 \% \mathrm{G} / \mathrm{C}$ and a $\mathrm{Tm}$ of $50 \%$ to $55^{\circ} \mathrm{C}$. Beaddisplayed probe libraries were produced by coupling oligonucleotide probes of interest to encoded microparticles ("beads") stained with combinations of fluorescent dyes. To produce a custom array composed of a desired set of specific probes, aliquots of corresponding beads were selected from the library, pooled, and dispensed onto silicon chips, and bonded on to a $1 \times 8$ chip carrier format shown in Fig. 1B. The resulting arrays exhibit a very high feature density, containing approximately 4000 beads of 32 or more spectrally distinguishable types within an area of $300 \times$ $300 \mu \mathrm{m}$. After array assembly, the color code of each bead within the array was recorded as a single snapshot (Fig. 1A) using an Array Imaging System (BAS). ${ }^{14}$

\section{Data analysis}

BAS's Image studio software suite was used to analyze fluorescence images and combine them with prerecorded decoding images showing the color codes for all beads within the array. Decoded image data were converted into CF results for Group I or Group II mutations and displayed as bar graphs (Fig. 2). For each allele of a given mutation, a normalized intensity (I) ratio represents the allelic ratio, namely $r=I_{A} / I_{B}=I_{A}-I_{N C} / I_{B}-I_{N C}$. where $I_{A}, I_{B}$, and $I_{N C}$ respectively denote: signal intensity for allele $A$, allele $\mathrm{B}$, and a negative control (NC).

In general, when the value of (I) was equal or less than zero, it was adjusted to 0.01 to avoid the generation of negative values. Allele discrimination was achieved by comparison of the fluorescent signals of beads containing the wild type and mutant probes. The result was calculated as the ratio of the wild type to mutant signal. The sample result is presented as the ratio of the signal of the wild type probe to the mutant probe. For most mutations, allelic ratios of $\geq 2.0$ were scored as homozygous for allele A, whereas an allelic ratio of $\leq 0.5$ was scored as homozygous for allele B. An allelic ratio of 0.5 to 2.0 was scored as heterozygous. Certain mutations including $711+1 \mathrm{G}>\mathrm{A}, \mathrm{R} 117 \mathrm{H}, \mathrm{G} 542 \mathrm{X}, \mathrm{R} 560 \mathrm{~T}$, and W1282X, required a heterozygous allelic ratio with an upper limit set at 2.50 . G551D and R334W required a heterozygous allelic ratio with an upper limit set at 3.00. These values were consistent for multiple runs, permitting reliable thresholding.

\section{RESULTS}

\section{Validation strategy}

The BeadChip assay system and eMAP protocol were validated using a panel of 26 CF mutations currently screened for in our laboratory including the ACMG 25 recommended mu- 
A
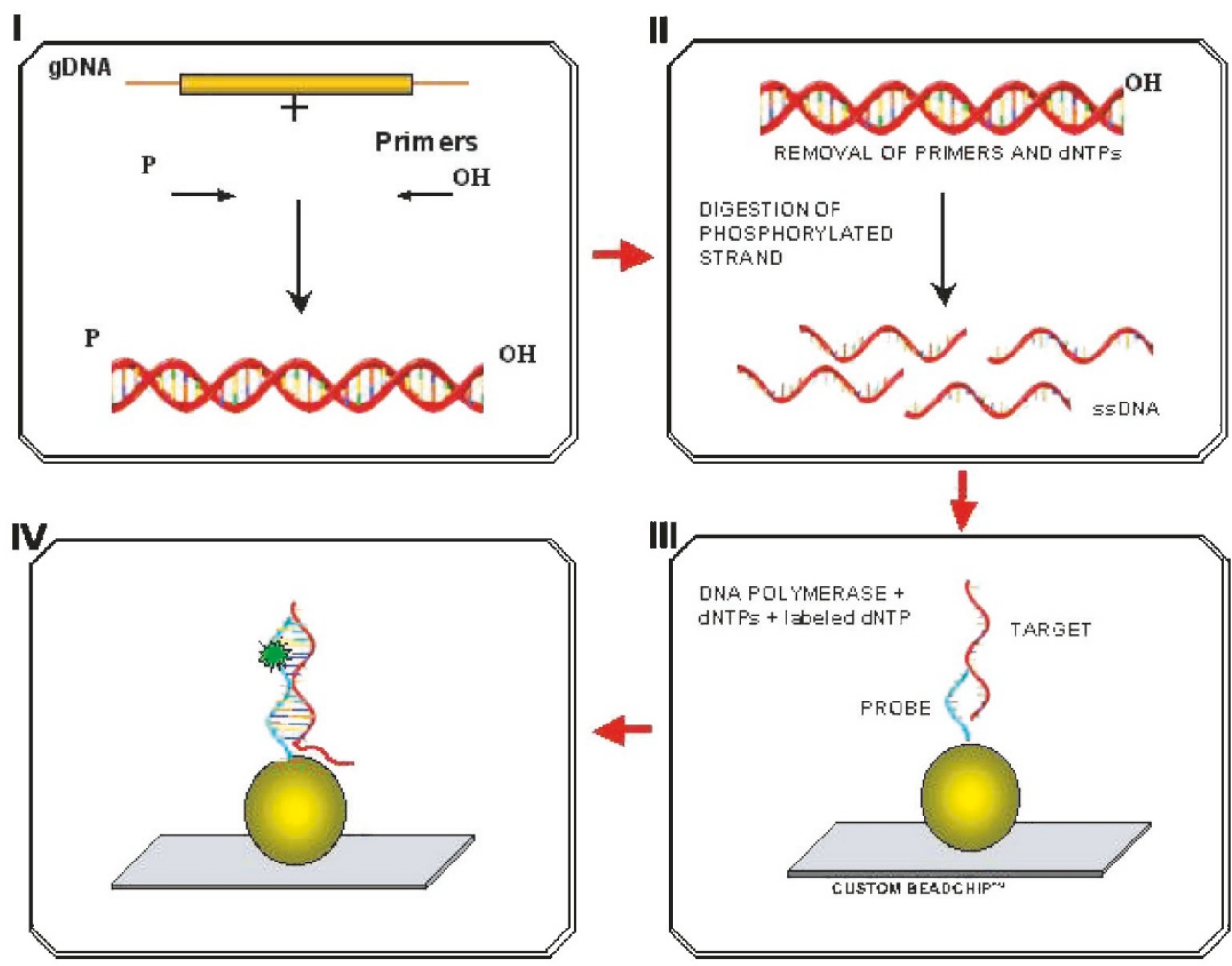

B

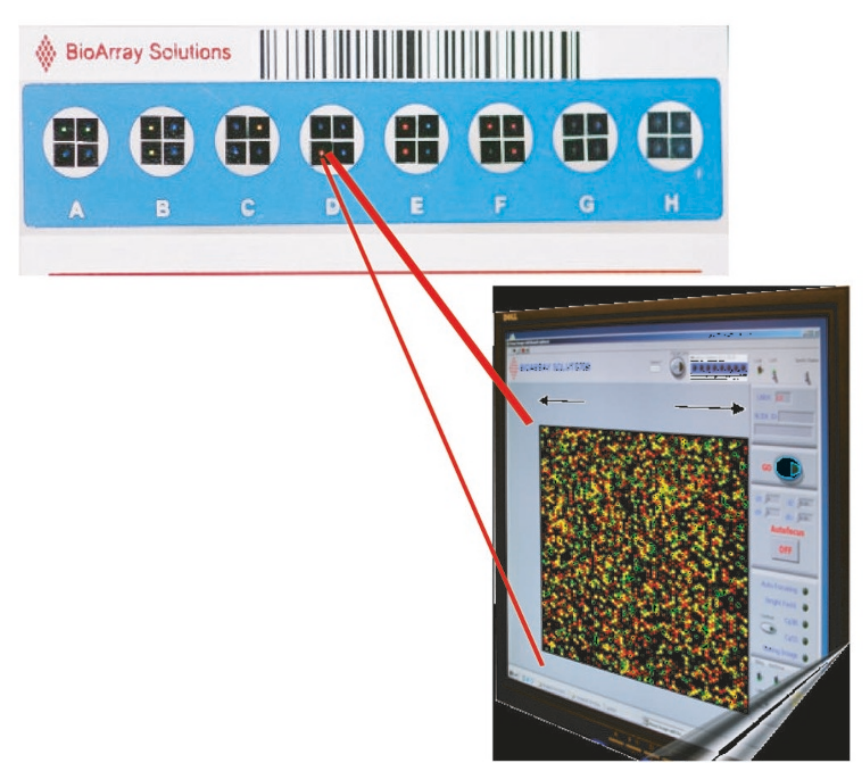

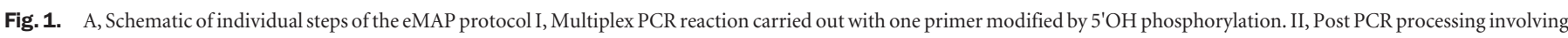

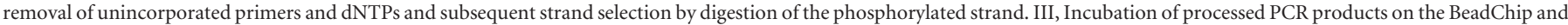

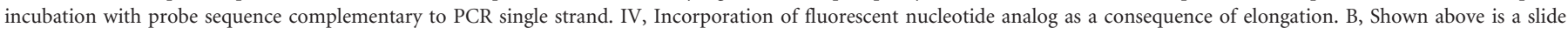

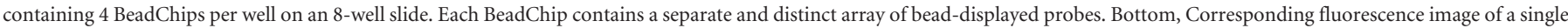
BeadChip as captured by the AIS is displayed, which represents the raw image data.

tations plus $\mathrm{D} 1152 \mathrm{H}$, a mutation that is prevalent in the $\mathrm{AJ}$ population. ${ }^{15,16}$ To assess the overall performance and feasibility of this technology for use in the genetic testing laboratory, we blindly assayed 507 patient samples, 12 proficiency sam- ples, and 145 control samples with the CF-26 BeadChip assay system and eMAP protocol, after reporting the testing results obtained by ASOH. The results are summarized in Table 1. The BeadChip assay system performed with high sensitivity and 


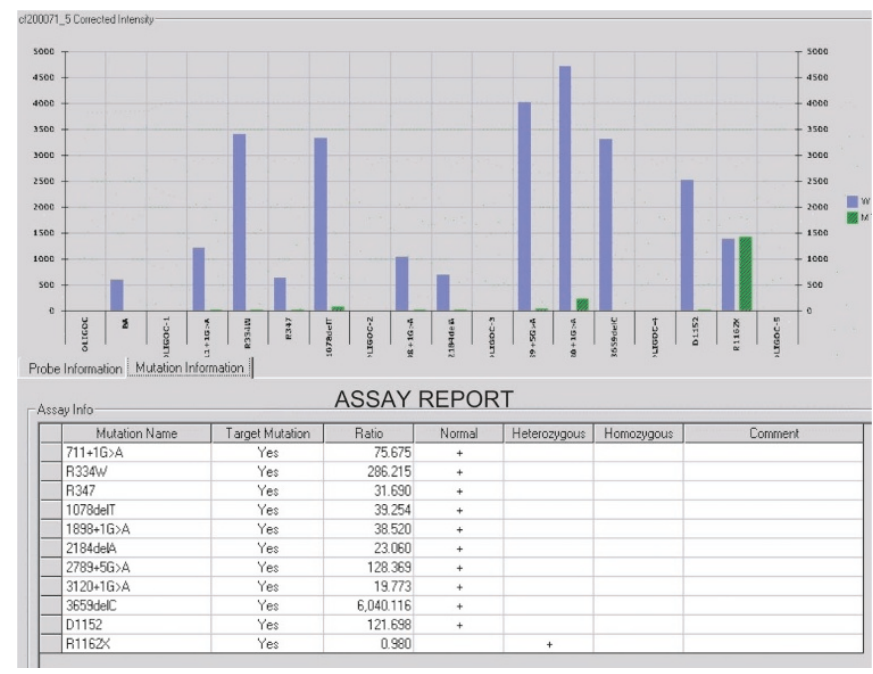

Fig. 2. Display ('snapshot') of CF using the BeadChip assay system. Shown are bar graphs of corrected signal intesities and corresponding results for each analyzed mutation in tabular format. Result of sample that was heterozygous for Group II mutation, R1162X.

accurately identified all 26 CF mutations, each of which was tested at least three times during the course of the validation. Assay performance was highly consistent among runs and failure rates were low and evenly distributed throughout the runs (I-VIII). The results obtained using the BeadChip assay system had $100 \%$ concordance with the results that were reported by our laboratory using the ASOH method.

The PCR failure rate for ASOH in our laboratory is $6.0 \%$, and primarily reflects the quality of the DNA samples, which are extracted by the simple and rapid lysis method. ${ }^{12}$ In contrast, the PCR failure rate for the BeadChip assay system was $3.5 \%$ (Table 1) representing samples in which at least one amplimer of the multiplex PCR reaction failed to amplify. Eighteen PCR failures were noted but in reality each sample had two separate PCRs and most samples were failures for one group but not the other. Although a failure of one group to amplify will delay reporting of the CF results, only a single reaction is repeated and the failure rate may be overestimated.

\section{Reflex testing}

With some methodologies, benign variants of codons 506, 507, and 508 in exon 10 can give false-positive results for the delF508 and delI507 mutations. Therefore, it is imperative to validate a new testing method for its ability to discriminate between these variant and mutant alleles. Genomic DNA from an F508C and an I506V carrier were amplified with the Group I multiplex primer mix and analyzed using the BeadChip assay system. In addition, single-stranded oligonucleotides for the I507V and $1506 \mathrm{M}$ variants, 40 nucleotides in length, were tested directly on Group I BeadChips. None of the variants produced fluorescent signals from delF508 or delI507 coupled beads indicating that no cross reactivity occurred with the mutant alleles. In addition, the PCR products amplified from the genomic DNA of F508C and I506V carriers and the singlestranded oligonucleotides for the I507V and I506M only elon- gated from the normal probe indicating that these variants did not interfere with allele discrimination. Thus, the eMAP design requires no separate reflex test to confirm benign variants.

Reflex or direct testing for the 5/7/9T polymorphic alleles in intron 8 was performed as a separate assay as recommended by the $\mathrm{ACMG}^{1}$ and was validated in this study. This reflex test is used for screenees who test positive for the $\mathrm{R} 117 \mathrm{H}$ mutation or are referred for male factor infertility and is performed by $\mathrm{ASOH}$ in our laboratory. Because the exon 9 amplimer in the Group I multiplex PCR reaction did not include the intron 8 polyT tract, an additional inclusive exon 9 amplification was performed and the products were analyzed using the eMAP protocol on PolyT BeadChips. Twelve samples were tested that included 5T, 7T, and 9T alleles and no discrepancies were found when compared with the prior results obtained using $\mathrm{ASOH}$.

\section{Assay performance}

The BeadChip assay system was used to analyze over 500 patient samples and 145 controls. The CF analysis program provided automatic allele calling and summarized results in two formats (Fig. 2). The corrected signal intensities, which have background signals subtracted, were presented as bar graphs with wild type and mutant signals for each mutation displayed adjacently. The results for each mutation were displayed in a table that provided allelic ratios and the corresponding result based on the ratios. The table also includes messages regarding the overall QC of the sample, any problems that occurred with the analysis and whether the sample should be repeated. Of the 519 samples tested, 500 were negative for the CF-26 panel and 19 were positive (3.7\%) (Table 1). Most importantly, there were no discrepancies between the results reported by our laboratory using the ASOH method and the results obtained using the BeadChip assay system.

Assay failure was scored as the inability to produce a result for at least one mutation on the BeadChip. Nine samples required a repeat analysis giving an assay failure rate of $1.7 \%$ (Table 1). As was noted above for PCR failures, this failure rate may be an overestimate as each sample was assayed for two separate groups of mutations and in all cases the failures were for a single group. The failure rates were spread evenly throughout the runs with each run, I-VIII, having 0,1 , or 2 failures with an average of 1.1 failures per run. Group II assays failed twice as often as Group I. In accordance with user selected settings, a low signal for any mutation (below 100) or high background prompted warning messages from the software and "flagged" samples were repeated. An allelic ratio (wild type to mutant) within 10\% of the preset threshold likewise prompted a warning message and the sample was repeated. This was more likely to happen when a wild-type sample had an allelic ratio that was close to the threshold of the heterozygote for that mutation.

Control samples were not factored into the failure rate, but one control sample failed analysis. A G551D/R553X compound heterozygote was reported as G551D homozygote, R553X heterozygote. This indicates that the R553X mutant 
Table 1

Data for runs performed with BeadChip technology

\begin{tabular}{|c|c|c|c|c|c|c|c|c|c|}
\hline Run no. & I & II & III & IV & $\mathrm{V}$ & VI & VII & VIII & Totals \\
\hline Samples tested & 87 & 57 & 69 & 72 & 66 & 35 & 72 & 61 & 519 \\
\hline Controls tested $^{k}$ & $0^{h}$ & $17^{h}$ & 20 & 29 & 22 & 16 & 20 & 21 & 145 \\
\hline PCR Failures ${ }^{i}$ & 4 & 4 & 2 & 1 & 1 & 2 & 1 & 3 & $18(3.5 \%)$ \\
\hline Assay Failures ${ }^{i}$ & 2 & 0 & 1 & 0 & 2 & 2 & 1 & 1 & $9(1.7 \%)$ \\
\hline Positives & $4^{a}$ & $3^{b}$ & 0 & $3^{c}$ & $4^{d}$ & $2^{e}$ & $2^{f}$ & $1^{g}$ & $19(3.7 \%)$ \\
\hline
\end{tabular}

${ }^{a} \mathrm{~W} 1282 \mathrm{X}$, delF508, D1152H, W1282X

${ }^{b}$ delF508, delF508, D1152H

cdelF508, R117H, R117H

${ }^{d} \mathrm{G} 542 \mathrm{X}$, delF508, D1152H, N1303K (does not include proficiency samples ${ }^{j}$ )

${ }^{e} \mathrm{~W} 1282 \mathrm{X}$, delF508

${ }_{\mathrm{I}} 148 \mathrm{~T}, 3849+10 \mathrm{kbC}>\mathrm{T}$

$8_{\mathrm{I} 148 \mathrm{~T}}$

${ }^{h}$ Runs I and II were amplified with the same master mix and used the same control samples.

${ }^{i}$ Failures are for either Group I or Group II and are for samples tested.

${ }^{j}$ Proficiency sample + s: delF508/G551D, R117H/delF508, R553X, delF508/delF508, 621+1G>T/delF508, delI507, delF508/3659delC, delF508/G551D

${ }^{k}$ Control samples were not included in calculation of failure rates. One control sample (G551D/R553X) failed in this study and is discussed in the text.

allele was not able to elongate from the G551 normal oligonucleotide, but the G551D mutant allele was able to elongate from the R553 normal oligonucleotide. This can be explained by examining the sequences of the probes and the mutant alleles. There is no mismatch between the G551D mutant allele and the R553 normal oligonucleotide because the mutation is outside the region of complementarity. However, a T:G mismatch exists between the R553M mutant allele and G551 normal oligonucleotide at the 6th position from the 3 ' end, which interrupts complementarity and elongation. For this combination of alleles, the assay system is therefore inaccurate. However, the reported genotype is unlikely and the sample would have been reflexed to another method for the analysis of the G551D and R553X mutations. This sample was originally sent to the laboratory as a CAP proficiency sample in 2001. The CAP summary of results stated that the sample was problematic for allele assignment with certain technologies. In our laboratory using ASOH, a signal was detected with the normal oligonucleotide as well as the mutant oligonucleotides for these two mutations, which made it difficult to determine whether the mutations were in cis or trans, and was ultimately resolved by sequencing. All other control samples, which included all 26 mutations in the heterozygous, compound heterozygous, or homozygous state, were identified correctly. Therefore, with one specific exception, the BeadChip assay system successfully identified all 26 mutations without ambiguity.

\section{DISCUSSION}

\section{Validation and comparison of assay techniques}

The BeadChip assay system offers several advantages over most if not all current $\mathrm{CF}$ testing methods. They include reduction in time and labor, reduction in PCR and assay repeats, automated allele calling, and electronic data storage. Using $\mathrm{ASOH}$, a single technician typically requires four days to complete CF screening for 26 mutations in two groups, permitting only weekly assays. The same technician using the BeadChip assay system can perform testing for up to 150 samples in only one day, which is a substantial reduction in time and labor. This difference should impact the way in which results are reported, such that sample repeats and confirmation of positive samples, which currently account for a two week turnaround time, would require only a single additional day. Therefore, results could be reported in less than one week and prenatal samples, which currently require at least three to four days for CF testing in our laboratory, would only take one day. In the diagnostic laboratory setting, this difference would also prompt changes in the structure of the work load such that new tests could easily be added without additional staff or the same tests would require a smaller staff effectively reducing labor costs.

With respect to performance, the BeadChip assay system had a lower PCR failure rate $(3.5 \%)$ than our current PCR strategy $(6.0 \%)$. These failure rates were within expected limits for the simple and rapid lysis method of DNA extraction currently used in the laboratory. ${ }^{11}$ The lower failure rate observed with the BeadChip assay system was most likely due to use of an initial "hot start" polymerase activation step, which results in increased enzyme specificity due to the 15 minutes denaturation step necessary for its activation.

The BeadChip assay failure rate was $1.7 \%$ and was fairly consistent from run to run. It is difficult to make a direct comparison to the ASOH because failures were scored by lack of signal from the control sample or high background on the filters, and were addressed by stripping and rehybridizing individual filters or by performing additional washes of filters, and did not require full repeat of the assay. However, it should be noted that these problems were common with the ASOH method and varied from week to week. In addition, often several different film exposures were required because of the multiple filters and probe groups that were used. Although a direct comparison of assay failure rates is difficult to make between 
the BeadChip assay and $\mathrm{ASOH}$, the former required less finetuning than $\mathrm{ASOH}$.

With respect to the other commercial technologies available for CF testing, the time required to perform the assay is comparable to the BeadChip assay system with ARMS, RBD, and OLA-PCR all requiring approximately one day for assay completion. ${ }^{9}$ The BeadChip assay required approximately seven hours for completion including PCR amplification. For testing of the CF-25 panel, an assay repeat rate of $1.9 \%$ for the RBD method using the Roche CF Gold LAp strip assay (Roche Molecular Systems) and 2.6\% for the OLA-PCR method using ABI instrumentation and allele calling software were reported. ${ }^{8}$ For the ARMS method, a repeat rate of $1.7 \%$ was observed using the CF20 Elucigene kit (Cellmark Diagnostics). ${ }^{17}$ The assay time requirements and repeat rate for the BeadChip assay system were therefore comparable to existing commercial technologies.

\section{Flexibility and limitations}

The CF-26 panel was developed at Bioarray Solutions, Ltd. specifically for custom use in our laboratory to mimic our current $\mathrm{CF}$ panel, which contains the $25 \mathrm{ABMG}$ recommended $\mathrm{CF}$ mutations and the D1152H AJ mutation. It is used for CF testing alone or as part of an AJ genetic testing panel. Three other versions of the CF panel were also created, CF-25, CF-38, and CF-50, all of which contain the core 25 with or without additional ethnic- or region-specific mutations. The current protocol for array production involves the assembly of libraries of individual ASO-coupled beads into arrays on demand and, therefore, specific customization is feasible. This level of flexibility allows the rapid addition or removal of mutations from a panel at will, requiring only a change in specification and software adjustment.

If not designed correctly, a potential limitation of elongation based assays is the interference of nondesignated polymorphisms present inpatient samples. Avoidance of known SNPs when designing elongation probes and PCR primers is necessary to minimize the occurrence of interference. In our eMAP design, all known CFTR SNPS were identified and potential conflicts on PCR primers and elongation probes were eliminated whenever possible. The problem encountered with the G551D/R553X control sample was the result of the R553X mutant strand failing to elongate from the G551 normal probe due to reduced annealing efficiency. Our results with the 506, 507, and 508 exon 10 variants indicate that they are capable of elongating from the normal F508 probe, but do not elongate from the mutant delF508 or delI507 probes.

The limits of the BeadChip technology are set by the limits of PCR multiplexing as well as the number of spectrally distinguishable beads. For example, BAS recently developed a PCR design for the CF-26 panel, which includes amplimers for all 16 exons in a single multiplex PCR reaction. Currently, 128 encoded bead types are available with the BeadChip assay system, which would allow for 64 different mutations to be assayed on a single BeadChip. The number can be further increased at least 4-fold by placing multiple BeadChips in a single well, which would require capture of separate fluorescence images from each BeadChip, but would allow for more mutations to be elongated simultaneously. The CF mutation panel does not push the upper limits of this technology. However, as new applications arise it will be interesting to explore the level of multiplexing possible using the eMAP approach.

In summary, a novel method for the analysis of CF mutations using BeadChip technology and eMAP protocol has been validated. The method was robust with exceptional discrimination of wild type and mutant alleles and had 100\% concordance with ASOH, a known reliable method of testing. The BeadChip technology offers flexibility and can be tailored to suit the needs of the patient population and is rapid such that PCR amplification and analysis can be performed in a single day. The system requires very little reflex testing as all mutations are analyzed directly and offers automated allele calling software and electronic data storage. The results of this validation support the conclusion that the BeadChip assay system provides an attractive and valid approach for the multiplexed analysis of CF mutations.

\section{ACKNOWLEDGMENTS}

This study was supported in part by a grant from the $\mathrm{Na}$ tional Center for Research Resources-NIH awarded to the Mount Sinai General Clinical Research Center (5 M01 RR00071) and a grant from BioArray Solutions, Ltd., Warren, New Jersey. The authors would like to thank Adebukola Olukoga for valuable technical assistance.

\section{References}

1. Grody WW, Cutting GR, Klinger KW, Richards CS, Watson MS, Desnick RJ. Laboratory standards and guidelines for population-based cystic fibrosis carrier screening. Genet Med 2001;3:149-154.

2. Richards CS, Bradley LA, Amos J, Allitto B, Grody WW, Maddalena A et al. Standards and Guidelines for CFTR Mutation Testing. Genet Med 2002;3:379391.

3. Hospital for Sick Children, Toronto, Cystic Fibrosis Mutation Database. Available at: http://www.genet.sickkids.on.ca/cftr/.

4. American College of Obstetrics and Gynecology, and American college of Hum Genet, Preconception and prenatal carrier screening for Cystic Fibrosis. Clinical and laboratory guidelines. Washington, DC: American College of Obstetrics and Gynecology, 2001.

5. Ranieri E, Ryall RG, Morris CP, Nelson PV, Carey WF, Pollard AC et al. Neonatal screening strategy for cystic fibrosis using immunoreactive trypsinogen and direct gene analysis. BMJ 1991;302:1237-1240.

6. Ferec C, Velingue C, Parent P, Morin JF, Codet JP, Rault G et al. Neonatal screening for cystic fibrosis: result of a pilot study using both immunoreactive trypsinogen and cystic fibrosis gene mutation analyses. Hum Genet 1995;96:542-548.

7. Gregg RG, Simantel A, Farrell PM, Koscik R, Kosorok MR, Laxova A et al. Newborn screening for cystic fibrosis in Wisconsin: Comparison of biochemical and molecular methods. Pediatrics 1997;99:819-824.

8. Strom CM, Huang D, Buller A, Redman J, Crossley B, Anderson B et al. Cystic fibrosis screening using the College Panel: platform comparison and lessons learned from the first 20,000 samples. Genet Med 2002;4:289-296.

9. Tomaiuolo R, Spina M, Castaldo G. Molecular diagnosis of cystic fibrosis: comparison of four analytical procedures. Clin Chem Lab Med 2003;41:26-32.

10. DeMarchi JM, Richards CS, Fenwick RG, Beaudet AL. A robotics-assisted procedure for large-scale cystic fibrosis mutation analysis. Hum Mutat 1994;4:281-290.

11. Higuchi R. Rapid efficient DNA extraction for PCR from cells or blood. Amplifications 1989;2:1-3.

12. Shuber AP, Grondin VJ, Klinger KL. A simplified procedure for developing multiplex PCRs. Gen Res 1995;5:488-493.

13. Shuber AP, Skoletsky J, Stern R, Handelin B. Efficient 12-mutation testing in the CFTR gene: General model for complex mutation analysis. Hum Mol Genet 1993;2: $153-158$. 


\section{Edelmann et al.}

14. Hashmi G, Song Y, Seul M, Edelmann L, Kornreich R, Desnick RJ. Analysis of cystic fibrosis and Ashkenazi Jewish disease mutations by multiplexed elongation of allele-specific oligonucleotides displayed on custom bead arrays. Am J Hum Gen 2002;71S:2316.

15. Feldmann D, Rochemaure J, Plouvier E, Magnier C, Chauve C, Aymard P. Mild course of cystic fibrosis in an adult with the D1152H mutation. Clin Chem 1995;41: 1675.
16. Kornreich K, Ekstein J, Edelmann L, Desnick, RJ. Premarital and Prenatal screening for cystic fibrosis: Experience in the Ashkenazi Jewish population. Genet Med 2004, 6:415-420.

17. Feldmann D, Guittard C, Georges MD, Houdayer C, Magnier C, Claustres M et al Genetic testing for cystic fibrosis: validation of the ELUCIGENE CF20 kit in blood and mouthwash samples. Ann Biol Clin 2001;59:277-283. 